TITLE:

\title{
Gauge-invariant formalism with a Dirac-mode expansion for confinement and chiral symmetry breaking
}

\section{$\operatorname{AUTHOR}(\mathrm{S})$ :}

Gongyo, Shinya; Iritani, Takumi; Suganuma Hideo

\section{CITATION:}

Gongyo, Shinya ... [et al]. Gauge-invariant formalism with a Dirac-mode expansion for confinement and chiral symmetry breaking. Physical Review D 2012, 86(3): 034510.

ISSUE DATE:

2012-08-31

URL:

http://hdl.handle.net/2433/198850

RIGHT:

(C) 2012 American Physical Society 
PHYSICAL REVIEW D 86, 034510 (2012)

\title{
Gauge-invariant formalism with a Dirac-mode expansion for confinement and chiral symmetry breaking
}

\author{
Shinya Gongyo, ${ }^{*}$ Takumi Iritani, ${ }^{\dagger}$ and Hideo Suganuma \\ Department of Physics, Graduate School of Science, Kyoto University, Kitashirakawa-Oiwakecho, Sakyo, Kyoto 606-8502, Japan
}

(Received 19 February 2012; published 31 August 2012)

\begin{abstract}
Using the eigenmode of the Dirac operator $\not D=\gamma^{\mu} D^{\mu}$ in quantum chromodynamics (QCD), we develop a manifestly gauge-covariant expansion and projection of the QCD operators such as the Wilson loop and the Polyakov loop. With this method, we perform a direct analysis of the correlation between confinement and chiral symmetry breaking in lattice QCD Monte Carlo calculation on $6^{4}$ at $\beta=5$.6. Even after removing the low-lying Dirac modes, which are responsible for chiral symmetry breaking, we find that the Wilson loop obeys the area law, and the slope parameter corresponding to the string tension, or confinement force, is almost unchanged. We find also that the Polyakov loop remains to be almost zero even without the low-lying Dirac modes, which indicates the $Z_{3}$-unbroken confinement phase. These results indicate that one-to-one correspondence does not hold between confinement and chiral symmetry breaking in QCD.
\end{abstract}

DOI: 10.1103/PhysRevD.86.034510

PACS numbers: 12.38.Gc, 12.38.Aw, 14.70.Dj

\section{INTRODUCTION}

These days, quantum chromodynamics (QCD) has been established as the fundamental gauge theory of the strong interaction. However, nonperturbative properties of lowenergy QCD such as color confinement and chiral symmetry breaking [1] are not yet well understood, which poses one of the most difficult problems in theoretical physics. The nonperturbative QCD has been studied in lattice QCD [2-6] and various analytical frameworks [7-12].

In particular, it is rather interesting and important to examine the correlation between confinement and chiral symmetry breaking [12-19], since a direct relation is not yet shown between them in QCD. A strong correlation between them has been suggested by the almost simultaneous phase transitions of deconfinement and chiral restoration in lattice QCD both at finite temperature $[5,20]$ and in a small-volume box [5].

The close relation between confinement and chiral symmetry breaking has also been suggested in terms of the monopole degrees of freedom [12-14]. Here, the monopole topologically appears in QCD by taking the maximally Abelian (MA) gauge [21-25]. For example, by removing the monopoles in the MA gauge, confinement and chiral symmetry breaking are simultaneously lost in lattice QCD $[13,14]$ (The instantons also disappear without monopoles [23]). This indicates an important role of the monopole to both confinement and chiral symmetry breaking, and these two nonperturbative QCD phenomena seem to be related via the monopole. However, as a possibility, removing the monopoles may be "too fatal" for most nonperturbative

\footnotetext{
*gongyo@ruby.scphys.kyoto-u.ac.jp

iritani@ruby.scphys.kyoto-u.ac.jp

suganuma@ruby.scphys.kyoto-u.ac.jp
}

properties. If this is the case, nonperturbative QCD phenomena are simultaneously lost by their cut.

In fact, there remains an important question: "if only the relevant ingredient of chiral symmetry breaking is carefully removed, how will confinement be in QCD?" Considering this question in this paper, we perform a direct investigation between color confinement and chiral symmetry breaking in lattice QCD, using the Dirac-mode expansion in a gauge-invariant manner [26].

The organization of this paper is as follows. In Sec. II, we introduce the gauge-invariant formalism with the Dirac-mode expansion. In Sec. III, we present the operator formalism in lattice QCD. In Sec. IV, we formulate the Dirac-mode expansion and projection. In Sec. V, we show the lattice results on the analysis of confinement in terms of the Dirac modes in QCD. Section VI is devoted to summary and discussions.

\section{GAUGE-INVARIANT FORMALISM WITH DIRAC-MODE EXPANSION}

We newly develop a manifestly gauge-covariant expansion of the QCD operator, e.g., the Wilson loop, using the eigenmode of the QCD Dirac operator $\not D=\gamma^{\mu} D^{\mu}$, and investigate the relation between confinement and chiral symmetry breaking.

\section{A. Gauge-covariant expansion in QCD instead of Fourier expansion}

In the previous studies [27,28], we investigated the relevant gluon momentum region for confinement in lattice $\mathrm{QCD}$, and found that the string tension $\sigma$, i.e., the confining force, is almost unchanged even after removing the high momentum gluon component above $1.5 \mathrm{GeV}$ in the Landau gauge. In fact, the confinement property originates from the low momentum gluon component below $1.5 \mathrm{GeV}$, which is the upper limit to contribute to $\sigma$. 
The previous study on the relevant gluonic modes was based on the Fourier expansion, i.e., the eigenmode expansion of the momentum operator $p^{\mu}$. Because of the commutable nature of $\left[p^{\mu}, p^{\nu}\right]=0$, all the momentum $p^{\mu}$ can be simultaneously diagonalized, which is one of the strong merits of the Fourier expansion. Also, it keeps Lorentz covariance.

However, the Fourier expansion does not keep gauge invariance in gauge theories. Therefore, for the use of the Fourier expansion in QCD, one has to select a suitable gauge such as the Landau gauge [27-29], where the gauge field fluctuation is strongly suppressed in Euclidean QCD.

As the next challenge, we consider a gauge-invariant method, using a gauge-covariant expansion in QCD instead of the Fourier expansion. In fact, we consider a generalization of the Fourier expansion or an alternative expansion with keeping the gauge symmetry.

A straight generalization is to use the covariant derivative operator $D^{\mu}$ instead of the derivative operator $\partial^{\mu}$. However, due to the noncommutable nature of $\left[D^{\mu}, D^{\nu}\right] \neq 0$, one cannot diagonalize all the covariant derivative $D^{\mu}(\mu=1$, $2,3,4)$ simultaneously, instead only one of them can be diagonalized. For example, the eigenmode expansion of $D_{4}$ keeps gauge covariance and is rather interesting, but this type of expansion inevitably breaks the Lorentz covariance. Thus, we consider the eigenmode expansion of the Dirac operator $\not D=\gamma^{\mu} D^{\mu}$ or $D^{2}=D^{\mu} D^{\mu}$ [30], since such an expansion keeps both gauge symmetry and Lorentz covariance.

In particular, the Dirac-mode expansion is rather interesting because the Dirac operator $\not D$ directly connects with chiral symmetry breaking via the Banks-Casher relation [8] and its zero modes are related to the topological charge via the Atiyah-Singer index theorem [31]. Here, we mainly consider the manifestly gauge-invariant new method using the Dirac-mode expansion. Thus, the Dirac-mode expansion has some important merits:

(i) The Dirac-mode expansion method manifestly keeps both gauge and Lorentz invariance.

(ii) Each QCD phenomenon can be directly investigated in terms of chiral symmetry breaking.

\section{B. Eigenmode of Dirac operator in lattice QCD}

Now, we consider the Dirac operator and its eigenmodes in lattice QCD formalism with spacing $a$ in the Euclidean metric. On the lattice, each site is labeled by $x=$ $\left(x_{1}, x_{2}, x_{3}, x_{4}\right)$ with $x_{\mu}$ being an integer. In lattice QCD, the gauge field is described by the link variable $U_{\mu}(x)=$ $e^{i a g A_{\mu}(x)} \in \mathrm{SU}\left(N_{c}\right)$, where $g$ is the QCD gauge coupling and $A_{\mu}(x) \in \operatorname{su}\left(N_{c}\right)$ corresponds to the gluon field.

In lattice QCD, the Dirac operator $\not D=\gamma_{\mu} D_{\mu}$ is expressed with $U_{\mu}(x)$ as

$$
\not D_{x, y}=\frac{1}{2 a} \sum_{\mu=1}^{4} \gamma_{\mu}\left[U_{\mu}(x) \delta_{x+\hat{\mu}, y}-U_{-\mu}(x) \delta_{x-\hat{\mu}, y}\right],
$$

where the convenient notation $U_{-\mu}(x) \equiv U_{\mu}^{\dagger}(x-\hat{\mu})$ is used. Here, $\hat{\mu}$ denotes the unit vector on the lattice in $\mu$-direction [5].

In this paper, we adopt the hermite definition of the $\gamma$-matrix, $\gamma_{\mu}^{\dagger}=\gamma_{\mu}$. Thus, $\not D$ is anti-hermite and satisfies

$$
\not D_{y, x}^{\dagger}=-\not D_{x, y} .
$$

The normalized eigenstate $|n\rangle$ of the Dirac operator $\not D$ is introduced as

$$
\not D|n\rangle=i \lambda_{n}|n\rangle,
$$

with $\lambda_{n} \in \mathbf{R}$. Because of $\left\{\gamma_{5}, \not \supset\right\}=0$, the state $\gamma_{5}|n\rangle$ is also an eigenstate of $\not D$ with the eigenvalue $-i \lambda_{n}$. The Dirac eigenfunction

$$
\psi_{n}(x) \equiv\langle x \mid n\rangle,
$$

obeys $\not \supset \psi_{n}(x)=i \lambda_{n} \psi_{n}(x)$, and its explicit form of the eigenvalue equation in lattice QCD is

$$
\begin{aligned}
& \frac{1}{2 a} \sum_{\mu=1}^{4} \gamma_{\mu}\left[U_{\mu}(x) \psi_{n}(x+\hat{\mu})-U_{-\mu}(x) \psi_{n}(x-\hat{\mu})\right] \\
& \quad=i \lambda_{n} \psi_{n}(x) .
\end{aligned}
$$

The Dirac eigenfunction $\psi_{n}(x)$ can be numerically obtained in lattice QCD apart from a phase factor.

According to $U_{\mu}(x) \rightarrow V(x) U_{\mu}(x) V^{\dagger}(x+\hat{\mu})$, the gauge transformation of $\psi_{n}(x)$ is found to be

$$
\psi_{n}(x) \rightarrow V(x) \psi_{n}(x),
$$

which is the same as that of the quark field. To be precise, for the Dirac eigenfunction, an irrelevant $n$-dependent global phase factor $e^{i \varphi_{n}[V]}$ appears, according to the arbitrariness of the definition of $\psi_{n}(x)$.

It is notable that the quark condensate $\langle\bar{q} q\rangle$, the order parameter of chiral symmetry breaking, is given by the zero eigenvalue density $\rho(0)$ of the Dirac operator, via the Banks-Casher relation [8],

$$
\langle\bar{q} q\rangle=-\lim _{m \rightarrow 0} \lim _{V \rightarrow \infty} \pi \rho(0) .
$$

Here, the spectral density of the Dirac operator is defined by

$$
\rho(\lambda) \equiv \frac{1}{V} \sum_{n}\left\langle\delta\left(\lambda-\lambda_{n}\right)\right\rangle,
$$

with the four-dimensional volume $V$. Also, the zero-mode number asymmetry of the Dirac operator $\not D$ is equal to the topological charge (the instanton number) $Q \equiv \frac{g^{2}}{16 \pi^{2}} \times$ $\int d^{4} x \operatorname{Tr}\left(G_{\mu \nu} \tilde{G}_{\mu \nu}\right)$, which is known as the Atiyah-Singer index theorem, Index $(\not D)=Q[31]$.

In calculating the eigenvalue of the Dirac operator $\not D$, we use the Kogut-Susskind (KS) formalism [3,5], which is often used to remove the redundant doublers of lattice fermions. Here, the use of the KS formalism is just the 
practical reason to reduce the calculation of the Dirac eigenvalues. In fact, the result of the Dirac-mode projection, which will be shown in Sec. IV, is unchanged, when the Dirac operator is directly diagonalized.

In the KS method, using $T(x) \equiv \gamma_{1}^{x_{1}} \gamma_{2}^{x_{2}} \gamma_{3}^{x_{3}} \gamma_{4}^{x_{4}}$ with $\gamma_{\mu}^{-k} \equiv\left(\gamma_{\mu}^{-1}\right)^{k}(k=1,2, \ldots)$, all the gamma matrices $\gamma_{\mu}$ are diagonalized as $T^{\dagger}(x) \gamma_{\mu} T(x \pm \hat{\mu})=\eta_{\mu}(x) 1$ with the staggered phase $\eta_{\mu}(x)$ defined by

$$
\eta_{1}(x) \equiv 1, \quad \eta_{\mu}(x)=(-1)^{x_{1}+\cdots+x_{\mu-1}}(\mu \geq 2) .
$$

For $\chi_{n}(x) \equiv T^{\dagger}(x) \psi_{n}(x)$, the Dirac eigenvalue equation has no spinor index, and the spinor degrees of freedom can be dropped off, which reduces the lattice-fermion species from 16 to 4 [5]. In the KS method, the Dirac operator $\gamma_{\mu} D_{\mu}$ is replaced by the KS Dirac operator $\eta_{\mu} D_{\mu}$,

$\left(\eta_{\mu} D_{\mu}\right)_{x, y}=\frac{1}{2 a} \sum_{\mu=1}^{4} \eta_{\mu}(x)\left[U_{\mu}(x) \delta_{x+\hat{\mu}, y}-U_{-\mu}(x) \delta_{x-\hat{\mu}, y}\right]$,

and the spinless eigenfunction $\chi_{n}(x)$ satisfies

$$
\begin{aligned}
& \frac{1}{2 a} \sum_{\mu=1}^{4} \eta_{\mu}(x)\left[U_{\mu}(x) \chi_{n}(x+\hat{\mu})-U_{-\mu}(x) \chi_{n}(x-\hat{\mu})\right] \\
& \quad=i \lambda_{n} \chi_{n}(x) .
\end{aligned}
$$

In the KS formalism, the chiral partner $\gamma_{5} \psi_{n}(x)$ reduces into $\eta_{5}(x) \chi_{n}(x)=(-1)^{x_{1}+x_{2}+x_{3}+x_{4}} \chi_{n}(x)$, which is an eigenfunction of $\eta_{\mu} D_{\mu}$ with the eigenvalue $-i \lambda_{n}$.

Using the KS formalism [3,5], the Dirac-mode number $L^{4} \times N_{c} \times 4$ is reduced to be $L^{4} \times N_{c}$ on the $L^{4}$ lattice. The actual number of the independent Dirac eigenvalue $\lambda_{n}$ is about $L^{4} \times N_{c} / 2$, due to the chiral property of the Dirac operator, i.e., pairwise appearance of $\pm \lambda_{n}$.

\section{OPERATOR FORMALISM IN LATTICE QCD}

To keep the gauge symmetry, careful treatments are necessary, since naive approximations may break the gauge symmetry. Here, we take the "operator formalism" [26], as explained below.

We define the link variable operator $\hat{U}_{ \pm \mu}$ by the matrix element of

$$
\left\langle x\left|\hat{U}_{ \pm \mu}\right| y\right\rangle=U_{ \pm \mu}(x) \delta_{x \pm \hat{\mu}, y} .
$$

Note that $\hat{U}_{\mu}$ and $\hat{U}_{-\mu}$ are Hermitian conjugate as the operator in Hilbert space in the sense that

$$
\begin{aligned}
\left\langle y\left|\hat{U}_{\mu}^{\dagger}\right| x\right\rangle & =U_{\mu}^{\dagger}(y) \delta_{y+\hat{\mu}, x}=U_{\mu}^{\dagger}(x-\hat{\mu}) \delta_{x-\hat{\mu}, y} \\
& =U_{-\mu}(x) \delta_{x-\hat{\mu}, y}=\left\langle x\left|\hat{U}_{-\mu}\right| y\right\rangle .
\end{aligned}
$$

In the operator formalism, Eq. (5) for the Dirac eigenstate is simply expressed as

$$
\frac{1}{2 a} \sum_{\mu=1}^{4} \gamma_{\mu}\left(\hat{U}_{\mu}-\hat{U}_{-\mu}\right)|n\rangle=i \lambda_{n}|n\rangle
$$

In the KS method, where the spinor index is dropped off, one identifies $\chi_{n}(x)=\langle x \mid n\rangle$, and then Eq. (11) for the KS Dirac eigenstate is expressed as

$$
\frac{1}{2 a} \sum_{\mu=1}^{4} \hat{\eta}_{\mu}\left(\hat{U}_{\mu}-\hat{U}_{-\mu}\right)|n\rangle=i \lambda_{n}|n\rangle
$$

where $\hat{\eta}_{\mu}$ is defined by $\left\langle x\left|\hat{\eta}_{\mu}\right| y\right\rangle=\eta_{\mu}(x) \delta_{x, y}$. Owing to $\eta_{\mu}(x \pm \hat{\mu})=\eta_{\mu}(x)$, one finds $\hat{\eta}_{\mu} \hat{U}_{ \pm \mu}=\hat{U}_{ \pm \mu} \hat{\eta}_{\mu}$, so that there is no ordering uncertainty in the KS Dirac operator in Eq. (15). In the KS method, the chiral partner $\gamma_{5}|n\rangle$ corresponds to $\hat{\eta}_{5}|n\rangle$, where $\hat{\eta}_{5}$ is defined by the matrix element $\left\langle x\left|\hat{\eta}_{5}\right| y\right\rangle=\eta_{5}(x) \delta_{x, y}=(-1)^{x_{1}+x_{2}+x_{3}+x_{4}} \delta_{x, y}$. Due to $\eta_{5}(x \pm \hat{\mu})=-\eta_{5}(x)$, we note $\hat{\eta}_{5} \hat{U}_{ \pm \mu}=-\hat{U}_{ \pm \mu} \hat{\eta}_{5}$.

In the following, we mainly use the ordinary Dirac operator $\gamma_{\mu} D_{\mu}$ and the spinor eigenfunction $\psi_{n}(x)=$ $\langle x \mid n\rangle$. When the KS method is applied, one only has to use the identification of $\chi_{n}(x)=\langle x \mid n\rangle$ in the following arguments. The final results are the same between both calculations based on $\gamma_{\mu} D_{\mu}$ and $\eta_{\mu} D_{\mu}$.

The Wilson loop operator $\hat{W}$ is defined as the product of $\hat{U}_{\mu}$ along a rectangular loop,

$$
\hat{W} \equiv \prod_{k=1}^{N} \hat{U}_{\mu_{k}}=\hat{U}_{\mu_{1}} \hat{U}_{\mu_{2}} \cdots \hat{U}_{\mu_{N}}
$$

For arbitrary loops, one finds $\sum_{k=1}^{N} \hat{\mu}_{k}=0$. We note that the functional trace of the Wilson loop operator $\hat{W}$ is proportional to the ordinary vacuum expectation value $\langle W\rangle$ of the Wilson loop:

$$
\begin{aligned}
\operatorname{Tr} \hat{W} & =\operatorname{tr} \sum_{x}\langle x|\hat{W}| x\rangle=\operatorname{tr} \sum_{x}\left\langle x\left|\hat{U}_{\mu_{1}} \hat{U}_{\mu_{2}} \cdots \hat{U}_{\mu_{N}}\right| x\right\rangle \\
& =\operatorname{tr} \sum_{x_{1}, x_{2}, \cdots, x_{N}}\left\langle x_{1}\left|\hat{U}_{\mu_{1}}\right| x_{2}\right\rangle\left\langle x_{2}\left|\hat{U}_{\mu_{2}}\right| x_{3}\right\rangle\left\langle x_{3}\left|\hat{U}_{\mu_{3}}\right| x_{4}\right\rangle \cdots\left\langle x_{N}\left|\hat{U}_{\mu_{N}}\right| x_{1}\right\rangle \\
& =\operatorname{tr} \sum_{x}\left\langle x\left|\hat{U}_{\mu_{1}}\right| x+\hat{\mu}_{1}\right\rangle\left\langle x+\hat{\mu}_{1}\left|\hat{U}_{\mu_{2}}\right| x+\sum_{k=1}^{2} \hat{\mu}_{k}\right\rangle \cdots\left\langle x+\sum_{k=1}^{N-1} \hat{\mu}_{k}\left|\hat{U}_{\mu_{N}}\right| x\right\rangle \\
& =\sum_{x} \operatorname{tr}\left\{U_{\mu_{1}}(x) U_{\mu_{2}}\left(x+\hat{\mu}_{1}\right) U_{\mu_{3}}\left(x+\sum_{k=1}^{2} \hat{\mu}_{k}\right) \cdots U_{\mu_{N}}\left(x+\sum_{k=1}^{N-1} \hat{\mu}_{k}\right)\right\}=\langle W\rangle \cdot \operatorname{Tr} 1 .
\end{aligned}
$$


Here, "Tr" denotes the functional trace, and "tr" denotes the trace over SU(3) color index.

The Dirac-mode matrix element of the link variable operator $\hat{U}_{\mu}$ can be expressed with $\psi_{n}(x)$ :

$$
\begin{aligned}
\langle m|\hat{U}| n\rangle & =\sum_{x}\langle m \mid x\rangle\left\langle x\left|\hat{U}_{\mu}\right| x+\hat{\mu}\right\rangle\langle x+\hat{\mu} \mid n\rangle \\
& =\sum_{x} \psi_{m}^{\dagger}(x) U_{\mu}(x) \psi_{n}(x+\hat{\mu}) .
\end{aligned}
$$

Although the total number of the matrix element is very huge, the matrix element is calculable and gauge-invariant, apart from an irrelevant phase factor. Using the gauge transformation (6), we find the gauge transformation of the matrix element as

$$
\begin{aligned}
\left\langle m\left|\hat{U}_{\mu}\right| n\right\rangle= & \sum_{x} \psi_{m}^{\dagger}(x) U_{\mu}(x) \psi_{n}(x+\hat{\mu}) \\
\rightarrow & \sum_{x} \psi_{m}^{\dagger}(x) V^{\dagger}(x) \cdot V(x) U_{\mu}(x) \\
& \times V^{\dagger}(x+\hat{\mu}) \cdot V(x+\hat{\mu}) \psi_{n}(x+\hat{\mu}) \\
= & \sum_{x} \psi_{m}^{\dagger}(x) U_{\mu}(x) \psi_{n}(x+\hat{\mu})=\left\langle m\left|\hat{U}_{\mu}\right| n\right\rangle .
\end{aligned}
$$

To be precise, an $n$-dependent global phase factor appears, corresponding to the arbitrariness of the phase in the basis $|n\rangle$. However, this phase factor cancels as $e^{-i \varphi_{n}} e^{i \varphi_{n}}=1$ between $|n\rangle$ and $\langle n|$, and does not appear for QCD physical quantities including the Wilson loop and the Polyakov loop.

In the practical lattice-QCD calculation, we adopt the KS formalism to reduce the computational complexity, as mentioned in Sec. II B. In the KS method, instead of $\psi_{n}(x)$, we use the spinless eigenfunction $\chi_{n}(x)$ of the KS Dirac operator $\eta_{\mu} D_{\mu}$, with the identification of $\chi_{n}(x)=\langle x \mid n\rangle$, and the KS-reduced matrix element of $\hat{U}_{\mu}$ is expressed as

$$
\begin{aligned}
\langle m|\hat{U}| n\rangle & =\sum_{x}\langle m \mid x\rangle\left\langle x\left|\hat{U}_{\mu}\right| x+\hat{\mu}\right\rangle\langle x+\hat{\mu} \mid n\rangle \\
& =\sum_{x} \chi_{m}^{\dagger}(x) U_{\mu}(x) \chi_{n}(x+\hat{\mu}) .
\end{aligned}
$$

In the arguments in the next section, the same results are obtained between the calculations based on the original Dirac operator $\gamma_{\mu} D_{\mu}$ and the KS Dirac operator $\eta_{\mu} D_{\mu}$.

\section{DIRAC-MODE EXPANSION AND PROJECTION}

\section{A. General definition of Dirac-mode expansion and projection}

From the completeness of the Dirac-mode basis, $\sum_{n}|n\rangle\langle n|=1$, we get

$$
\hat{O}=\sum_{m} \sum_{n}|m\rangle\langle m|\hat{O}| n\rangle\langle n|,
$$

for arbitrary operators. Based on this relation, the Diracmode expansion and projection can be defined [26]. We define the projection operator $\hat{P}$ which restricts the Diracmode space,

$$
\hat{P} \equiv \sum_{n \in A}|n\rangle\langle n|,
$$

where $A$ denotes an arbitrary set of Dirac modes. In $\hat{P}$, the arbitrary phase cancels between $|n\rangle$ and $\langle n|$. One finds $\hat{P}^{2}=\hat{P}$ and $\hat{P}^{\dagger}=\hat{P}$. The typical projections are IR cut and UV cut of the Dirac modes:

$$
\hat{P}_{\mathrm{IR}} \equiv \sum_{\left|\lambda_{n}\right| \geq \Lambda_{\mathrm{IR}}}|n\rangle\left\langle n\left|, \quad \hat{P}_{\mathrm{UV}} \equiv \sum_{\left|\lambda_{n}\right| \leq \Lambda_{\mathrm{UV}}}\right| n\right\rangle\langle n| .
$$

Using the projection operator $\hat{P}$, we define the Diracmode projected link variable operator,

$$
\hat{U}_{\mu}^{P} \equiv \hat{P} \hat{U}_{\mu} \hat{P}=\sum_{m \in A} \sum_{n \in A}|m\rangle\left\langle m\left|\hat{U}_{\mu}\right| n\right\rangle\langle n| .
$$

During this projection, there appears to be some nonlocality in general, but it would not be important for the argument of large-distance properties such as confinement.

Each lattice QCD configuration is characterized by the set of the link variable $\left\{U_{\mu}(s)\right\}$, or equivalently, the link variable operator $\left\{\hat{U}_{\mu}\right\}$, and then the Dirac-mode projection is described by the replacement of $\left\{\hat{U}_{\mu}\right\}$ by $\left\{\hat{U}_{\mu}^{P}\right\}$. In fact, the Dirac-mode projection of QCD physical quantities $\left\langle O\left[U_{\mu}(s)\right]\right\rangle$ or $\operatorname{Tr} \hat{O}\left[\hat{U}_{\mu}\right]$ can be defined by the replacement of

$$
\operatorname{Tr} \hat{O}\left[\hat{U}_{\mu}\right] \rightarrow \operatorname{Tr} \hat{O}\left[\hat{U}_{\mu}^{P}\right] .
$$

Also in full QCD, after the integration over the quark degrees of freedom, all the QCD physical quantities can be written by $\left\langle O\left[U_{\mu}(s)\right]\right\rangle$ or $\operatorname{Tr} \hat{O}\left[\hat{U}_{\mu}\right]$, so that the Diracmode projection can be applied in the same way.

\section{B. Dirac-mode expansion and projection of the Wilson loop}

In this subsection, we consider the Dirac-mode expansion and projection of the Wilson loop $\langle W(R, T)\rangle \propto$ $\operatorname{Tr} \hat{W}(R, T)$ corresponding to the $R \times T$ rectangular loop.

For the ordinary Wilson loop $\langle W(R, T)\rangle$, its area law indicates the confinement phase of the QCD vacuum and the linear arising potential between static quark and antiquark in the infrared region [5].

From the Wilson loop operator $\hat{W} \equiv \prod_{k=1}^{N} \hat{U}_{\mu_{k}}$, we get the Dirac-mode expansion of the Wilson loop as

$$
\begin{aligned}
\operatorname{Tr} \hat{W} & =\operatorname{Tr} \prod_{k=1}^{N} \hat{U}_{\mu_{k}}=\operatorname{Tr}\left(\hat{U}_{\mu_{1}} \hat{U}_{\mu_{2}} \cdots \hat{U}_{\mu_{N}}\right) \\
& =\operatorname{tr} \sum_{n_{1}, n_{2}, \cdots, n_{N}}\left\langle n_{1}\left|\hat{U}_{\mu_{1}}\right| n_{2}\right\rangle\left\langle n_{2}\left|\hat{U}_{\mu_{2}}\right| n_{3}\right\rangle \cdots\left\langle n_{N}\left|\hat{U}_{\mu_{N}}\right| n_{1}\right\rangle .
\end{aligned}
$$

Based on this expression, we investigate the role of specific Dirac modes on the area law of the Wilson loop. In fact, if 
some Dirac modes are essential to reproduce the area law of the Wilson loop or the confinement property, the removal of the coupling to these modes leads to a significant change on the area law.

In this way, we try to answer the question: "Are there any relevant Dirac modes responsible to the area law of the Wilson loop?"

To this end, we define the Dirac-mode projected Wilson loop operator,

$$
\begin{aligned}
\hat{W}^{P} \equiv & \prod_{k=1}^{N} \hat{U}_{\mu_{k}}^{P}=\hat{U}_{\mu_{1}}^{P} \hat{U}_{\mu_{2}}^{P} \cdots \hat{U}_{\mu_{N}}^{P} \\
= & \hat{P} \hat{U}_{\mu_{1}} \hat{P} \hat{U}_{\mu_{2}} \hat{P} \cdots \hat{P} \hat{U}_{\mu_{N}} \hat{P} \\
= & \sum_{n_{1}, n_{2}, \cdots, n_{N+1} \in A}\left|n_{1}\right\rangle\left\langle n_{1}\left|\hat{U}_{\mu_{1}}\right| n_{2}\right\rangle \\
& \times\left\langle n_{2}\left|\hat{U}_{\mu_{2}}\right| n_{3}\right\rangle \cdots\left\langle n_{N}\left|\hat{U}_{\mu_{N}}\right| n_{N+1}\right\rangle\left\langle n_{N+1}\right| .
\end{aligned}
$$

Then, we obtain the functional trace of the Dirac-mode projected Wilson loop operator,

$$
\begin{aligned}
\operatorname{Tr} \hat{W}^{P}= & \operatorname{Tr} \prod_{k=1}^{N} \hat{U}_{\mu_{k}}^{P}=\operatorname{Tr} \hat{U}_{\mu_{1}}^{P} \hat{U}_{\mu_{2}}^{P} \cdots \hat{U}_{\mu_{N}}^{P} \\
= & \operatorname{Tr} \hat{P} \hat{U_{\mu_{1}}} \hat{P} \hat{U}_{\mu_{2}} \hat{P} \cdots \hat{P} \hat{U}_{\mu_{N}} \hat{P} \\
= & \operatorname{tr} \sum_{n_{1}, n_{2}, \cdots, n_{N} \in A}\left\langle n_{1}\left|\hat{U}_{\mu_{1}}\right| n_{2}\right\rangle \\
& \times\left\langle n_{2}\left|\hat{U}_{\mu_{2}}\right| n_{3}\right\rangle \cdots\left\langle n_{N}\left|\hat{U}_{\mu_{N}}\right| n_{1}\right\rangle,
\end{aligned}
$$

which is manifestly gauge invariant. Here, the arbitrary phase factor cancels between $\left|n_{k}\right\rangle$ and $\left\langle n_{k}\right|$. Its gauge invariance is also numerically checked in the lattice QCD Monte Carlo calculation.

The original Wilson loop operator $\hat{W}(R, T)$ couples to all the Dirac modes, and $\operatorname{Tr} \hat{W}(R, T)$ obeys the area law,

$$
\operatorname{Tr} \hat{W}(R, T) \propto\langle W(R, T)\rangle \propto e^{-\sigma R T},
$$

for large $R$ and $T$. Here, the slope parameter $\sigma$ corresponds to the string tension, or confinement force. For the restriction of the Dirac-mode space to be $A$, we investigate the Dirac-mode projected Wilson loop operator $\hat{W}^{P}(R, T)$, which couples to the restricted Dirac modes. If the removed Dirac modes are essential for the confinement property or the area-law behavior of the Wilson loop, a large change is expected on the behavior of $\operatorname{Tr} \hat{W}^{P}(R, T)$. If not, no significant change is expected on the behavior of $\operatorname{Tr} \hat{W}^{P}(R, T)$. In fact, one can investigate the role of the removed Dirac modes to confinement by checking the area-law behavior of $\operatorname{Tr} \hat{W}^{P}(R, T)$ and the slope parameter $\sigma^{P}$, which is formally written as

$$
\sigma^{P} \equiv-\lim _{R, T \rightarrow \infty} \frac{1}{R T} \ln \left\{\operatorname{Tr} \hat{W}^{P}(R, T)\right\} .
$$

\section{Corresponding Dirac-mode projected interquark potential}

For the estimation of the slope parameter $\sigma^{P}$ from $\operatorname{Tr} \hat{W}^{P}(R, T)$, we define the corresponding Dirac-mode projected interquark potential,

$$
V^{P}(R) \equiv-\lim _{T \rightarrow \infty} \frac{1}{T} \ln \left\{\operatorname{Tr} \hat{W}^{P}(R, T)\right\},
$$

which is also manifestly gauge invariant. To be precise, because of the nonlocality appearing in the Dirac-mode projection, $V^{P}(R)$ does not have a definite meaning of the static potential. However, it is still useful to obtain $\sigma^{P}$ in Eq. (30) from $\operatorname{Tr} \hat{W}^{P}(R, T)$. In fact, $\sigma^{P}$ is obtained from the infrared slope of $V^{P}(R)$. Note also that, in the unprojected case of $\hat{P}=1$, the ordinary interquark potential is obtained apart from an irrelevant constant,

$$
\begin{aligned}
V(R) & =-\lim _{T \rightarrow \infty} \frac{1}{T} \ln \{\operatorname{Tr} \hat{W}(R, T)\} \\
& =-\lim _{T \rightarrow \infty} \frac{1}{T} \ln \langle W(R, T)\rangle+\text { irrelevant const, }
\end{aligned}
$$

because of $\operatorname{Tr} \hat{W}=\langle W\rangle \cdot \operatorname{Tr} 1$, as was derived in Eq. (17).

\section{ANALYSIS OF CONFINEMENT IN TERMS OF DIRAC MODES IN QCD}

We consider various projection space $A$ in the Dirac-mode space, e.g., IR cut or UV cut of Dirac modes. With this Dirac-mode expansion and projection formalism, we calculate the Dirac-mode projected Wilson loop $\operatorname{Tr} W^{P}(R, T)$ in a gauge-invariant manner. In particular, using IR cut of the Dirac modes, we directly investigate the relation between chiral symmetry breaking and confinement as the area-law behavior of the Wilson loop, since the low-lying Dirac modes are responsible to chiral symmetry breaking.

As a technical difficulty of this formalism, we have to deal with huge dimensional matrices and their products. Actually, the total matrix dimension of $\left\langle m\left|\hat{U}_{\mu}\right| n\right\rangle$ is (Dirac-modenumber) ${ }^{2}$. On the $L^{4}$ lattice, the Dirac-mode number is $L^{4} \times N_{c} \times 4$, which can be reduced to be $L^{4} \times N_{c}$, using the $\mathrm{KS}$ formalism [3,5], as mentioned in Sec. II B. The actual number of the independent Dirac eigenvalue $\lambda_{n}$ is about $L^{4} \times N_{c} / 2$, due to the chiral property of $\not D$, i.e., pairwise appearance of $\pm \lambda_{n}$. Even for the projected operators, where the Dirac-mode space is restricted, the matrix is generally still huge. In addition, we have to deal with the product of the huge matrices $\left\langle m\left|\hat{U}_{\mu}\right| n\right\rangle$ in calculating the Wilson loop. Thus, at present, we use a small-size lattice in the numerical calculation.

In this paper, we perform the SU(3) lattice QCD Monte Carlo calculation with the standard plaquette action at $\beta=$ 5.6 on $6^{4}$ at the quenched level, using the pseudo-heat-bath algorithm. The ordinary periodic boundary condition is used for the link variable. The gauge configurations are 
taken every 500 sweeps after 10,000 sweeps thermalization, and 20 gauge configurations are used for each analysis. At $\beta=5.6$, the lattice spacing $a$ is estimated as $a \simeq 0.25 \mathrm{fm}$, i.e., $a^{-1} \simeq 0.8 \mathrm{GeV}$, which leads to the string tension $\sigma \simeq 0.89 \mathrm{GeV} / \mathrm{fm}$ in the interquark potential (This estimate is done also on a larger volume lattice). Then, the total volume is $V=(6 a)^{4} \simeq(1.5 \mathrm{fm})^{4}$, and the momentum cutoff is $\pi / a \simeq 2.5 \mathrm{GeV}$.

On the $6^{4}$ lattice, the Dirac-mode number is $6^{4} \times 3 \times$ $4=15,552$, which is reduced to $6^{4} \times 3=3,888$ using the KS formalism. In fact, the KS Dirac operator $\eta_{\mu} D_{\mu}$ and the KS-reduced matrix element $\left\langle m\left|\hat{U}_{\mu}\right| n\right\rangle$ are expressed by $3,888 \times 3,888$ matrix. Considering the pairwise appearance of $\lambda_{n}$ and $-\lambda_{n}$, the actual number of the independent Dirac eigenvalue $\lambda_{n}$ is reduced to be around $6^{4} \times 3 / 2=$ $1,944$.

To diagonalize the KS Dirac operator $\eta_{\mu} D_{\mu}$, we use Linear Algebra PACKage (LAPACK) [32]. For the statistical error on the lattice data, we adopt the jackknife error estimate [5].

We show in Fig. 1(a) the spectral density $\rho(\lambda)$ of the QCD Dirac operator $\not D$. The chiral property of $\not D$ leads to $\rho(-\lambda)=\rho(\lambda)$. Figure 1(b) is the IR-cut Dirac spectral density

$$
\rho_{\mathrm{IR}}(\lambda) \equiv \rho(\lambda) \theta\left(|\lambda|-\Lambda_{\mathrm{IR}}\right)
$$

with the IR cutoff $\Lambda_{\mathrm{IR}}=0.5 a^{-1} \simeq 0.4 \mathrm{GeV}$.

Note that, using the eigenvalue $\lambda_{n}$, the quark condensate $\langle\bar{q} q\rangle$ is obtained as

$$
\begin{aligned}
\langle\bar{q} q\rangle & =-\frac{1}{V} \operatorname{Tr} \frac{1}{\not D+m}=-\frac{1}{V} \sum_{n} \frac{1}{i \lambda_{n}+m} \\
& =-\frac{1}{V}\left(\sum_{\lambda_{n}>0} \frac{2 m}{\lambda_{n}^{2}+m^{2}}+\frac{\nu}{m}\right),
\end{aligned}
$$

where $\nu$ is the total number of the zero mode of $\not D$. Here, the nonzero eigenvalues appear as pairwise, which makes $\langle\bar{q} q\rangle$ real. (In lattice QCD, one has to take account of the doubler contribution, which can be regarded as flavor at the quenched level.) Then, in the presence of the IR cut $\Lambda_{\mathrm{IR}}$ for the Dirac eigenmode, the quark condensate is obtained as

$$
\langle\bar{q} q\rangle_{\Lambda_{\mathrm{IR}}}=-\frac{1}{V} \sum_{\lambda_{n} \geq \Lambda_{\mathrm{IR}}} \frac{2 m}{\lambda_{n}^{2}+m^{2}} .
$$

We show in Fig. 2 the lattice QCD result of the quark condensate $\langle\bar{q} q\rangle_{\Lambda_{\mathrm{IR}}}$ as a function of the current quark mass $m$ in the presence of IR cut $\Lambda_{\mathrm{IR}}$.

By removing the low-lying Dirac modes, the chiral condensate $\langle\bar{q} q\rangle$ is largely reduced, reflecting the BanksCasher relation. Actually, directly from lattice QCD calculation, we find a large reduction of the chiral condensate in the presence of the IR cut $\Lambda_{\mathrm{IR}}=0.5 a^{-1} \simeq 0.4 \mathrm{GeV}$,

$$
\frac{\langle\bar{q} q\rangle_{\Lambda_{\mathrm{IR}}}}{\langle\bar{q} q\rangle} \simeq 0.02 \text {, }
$$
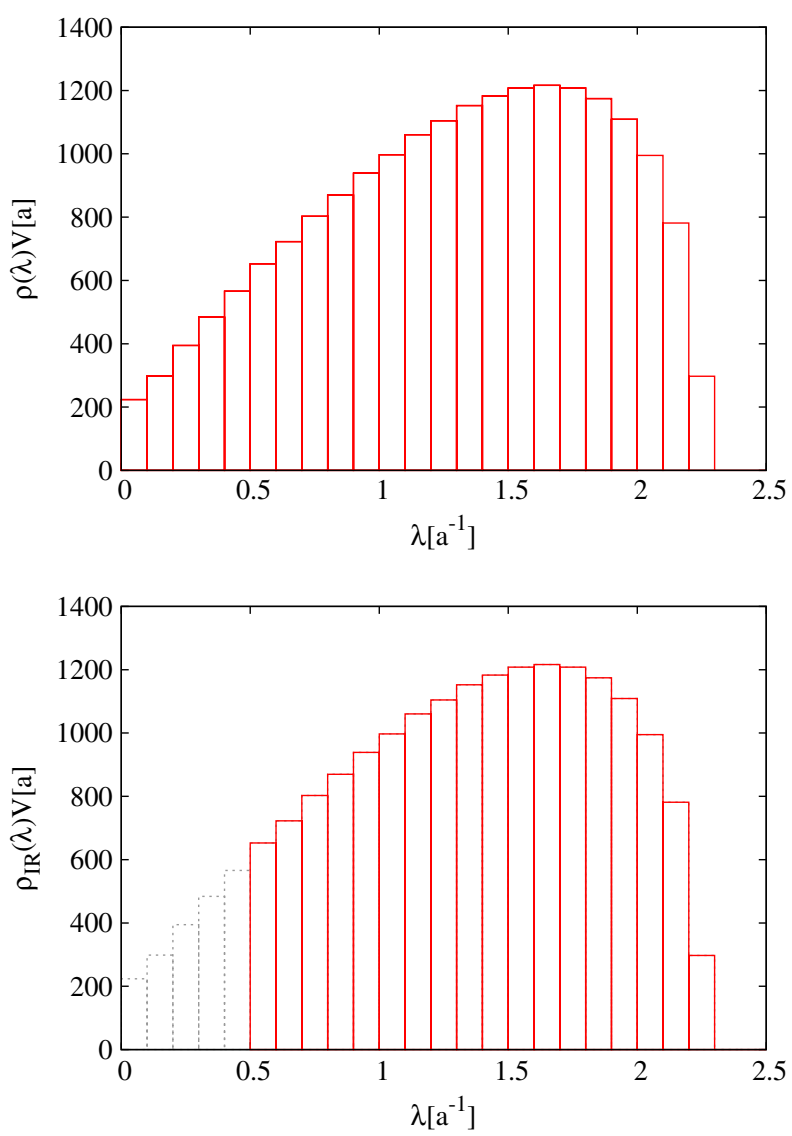

FIG. 1 (color online). (a) The spectral density $\rho(\lambda)$ of the Dirac operator in lattice QCD at $\beta=5.6$ and $6^{4}$. The negative- $\lambda$ region is omitted, because of $\rho(-\lambda)=\rho(\lambda)$. The volume $V$ is multiplied. (b) The IR-cut Dirac spectral density $\rho_{\mathrm{IR}}(\lambda) \equiv \rho(\lambda) \theta\left(|\lambda|-\Lambda_{\mathrm{IR}}\right)$ with the IR cutoff $\Lambda_{\mathrm{IR}}=0.5 a^{-1} \simeq$ $0.4 \mathrm{GeV}$.

around the physical region of $m \simeq 0.006 a^{-1} \simeq 5 \mathrm{MeV}$ [33], as shown in Fig. 2.

Now, let us consider the removal of the coupling to the low-lying Dirac modes from the Wilson loop $\langle W(R, T)\rangle$. Figure 3 shows the Dirac-mode projected Wilson loop $\left\langle W^{P}(R, T)\right\rangle \equiv \operatorname{Tr} \hat{W}^{P}(R, T)$ after removing low-lying Dirac modes, which is obtained in lattice QCD with the IR cut of $\rho_{\text {IR }}(\lambda) \equiv \rho(\lambda) \theta\left(|\lambda|-\Lambda_{\text {IR }}\right)$ with the IR cutoff $\Lambda_{\mathrm{IR}}=0.5 a^{-1}$. Even after removing the coupling to the low-lying Dirac modes, which are responsible to chiral symmetry breaking, the Dirac-mode projected Wilson loop is found to obey the area law as

$$
\left\langle W^{P}(R, T)\right\rangle \propto e^{-\sigma^{P} R T},
$$

and the slope parameter $\sigma^{P}$ corresponding to the string tension, or confinement force, is almost unchanged as

$$
\sigma^{P} \simeq \sigma
$$

In fact, the confinement property seems to be kept in the absence of the low-lying Dirac modes or the essence of 


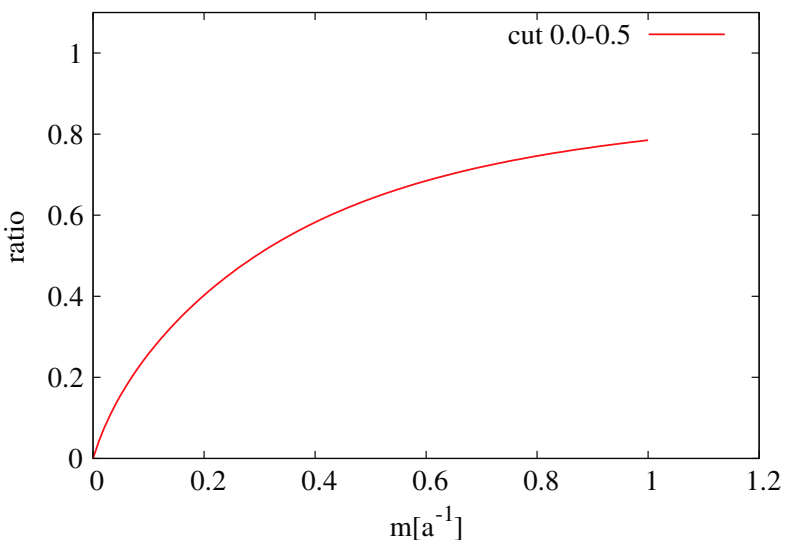

FIG. 2 (color online). The lattice QCD result of the quark condensate $\langle\bar{q} q\rangle_{\Lambda_{\mathrm{IR}}}$ as a function of the current quark mass $m$ in the presence of IR cut $\Lambda_{\mathrm{IR}}=0.5 a^{-1}$. The vertical axis is normalized by the original value of $\langle\bar{q} q\rangle$ without cut. A large reduction is found as $\langle\bar{q} q\rangle_{\Lambda_{\mathrm{IR}}} /\langle\bar{q} q\rangle \simeq 0.02$ for $\Lambda_{\mathrm{IR}}=0.5 a^{-1} \simeq$ $0.4 \mathrm{GeV}$ around the physical region of $m \simeq 0.006 a^{-1} \simeq 5 \mathrm{MeV}$.

chiral symmetry breaking [26]. This result indicates that one-to-one correspondence does not hold for confinement and chiral symmetry breaking in QCD.

Next, to estimate the slope parameter $\sigma^{P}$, we consider the potential $V^{P}(R)$ obtained from $\left\langle W^{P}(R, T)\right\rangle$. Figure 4 shows the "effective mass" of the interquark potential $V_{\text {eff }}(R, T) \equiv \ln \left[\left\langle W^{P}(R, T)\right\rangle /\left\langle W^{P}(R, T+1)\right\rangle\right]$ after removing the low-lying Dirac modes, plotted against $T$ at each $R$. One finds the "plateau" or the stability of the effective mass $V_{\text {eff }}(R, T)$ against $T$, which means the dominance of the ground-state component. Similarly, in the standard

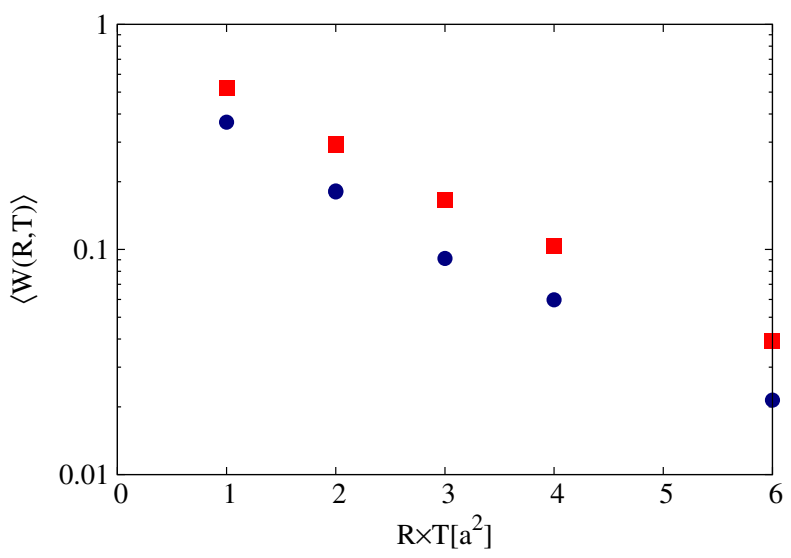

FIG. 3 (color online). The lattice QCD result of the Diracmode projected Wilson loop $\left\langle W^{P}(R, T)\right\rangle \equiv \operatorname{Tr} \hat{W}^{P}(R, T)$ after removing low-lying Dirac modes, plotted against the area $R \times$ $T$. Circles denote the Wilson loop obtained with the IR cut of $\rho_{\mathrm{IR}}(\lambda) \equiv \rho(\lambda) \theta\left(|\lambda|-\Lambda_{\mathrm{IR}}\right)$ with the IR cutoff $\Lambda_{\mathrm{IR}}=0.5 a^{-1}$. Squares denote the original Wilson loop $\langle W(R, T)\rangle$. $\left\langle W^{P}(R, T)\right\rangle$ seems to obey the area law with the same slope parameter, $\sigma^{P} \simeq \sigma$.

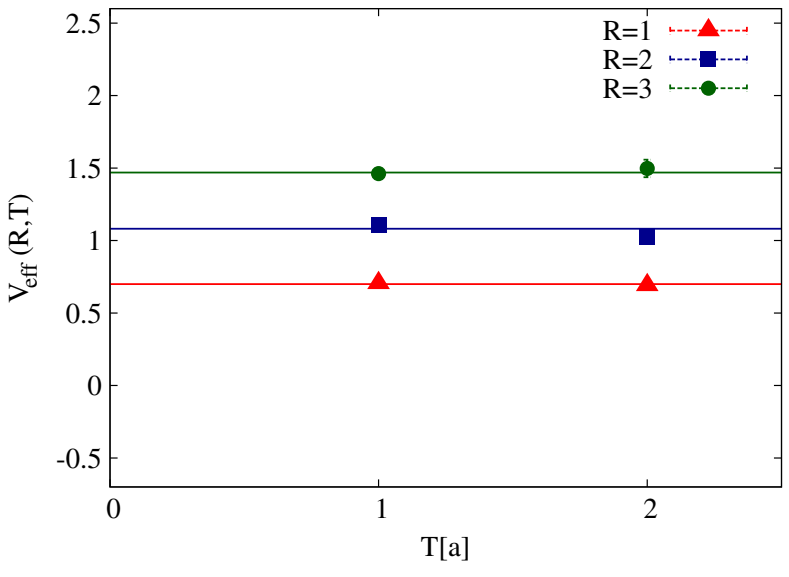

FIG. 4 (color online). The effective mass plot of the interquark potential $V_{\text {eff }}(R, T) \equiv \ln \left[\left\langle W^{P}(R, T)\right\rangle /\left\langle W^{P}(R, T+1)\right\rangle\right]$ after removing the low-lying Dirac modes. $V_{\text {eff }}(R, T)$ is plotted against $T$ for each $R$. The horizontal lines denote the best-fit value in the exponential fit of Eq (39) at each $R$.

procedure to obtain potentials in lattice QCD [5,34], we determine the interquark potential $V^{P}(R)$ by the exponential fit of the Wilson loop

$$
\left\langle W^{P}(R, T)\right\rangle=C e^{-V^{P}(R) T}
$$

for $T=1,2,3$, which corresponds to the plateau region of $T=1,2$ in $V_{\text {eff }}(R, T)$. Figure 5 shows the Dirac-mode projected interquark potential $V^{P}(R)$ after removing lowlying Dirac modes below the IR cutoff $\Lambda_{\mathrm{IR}}=0.5 a^{-1}$. No significant change is observed on the interquark potential besides an irrelevant constant, that is, the slope parameter $\sigma^{P}$ is almost unchanged, even after removing the low-lying Dirac modes.

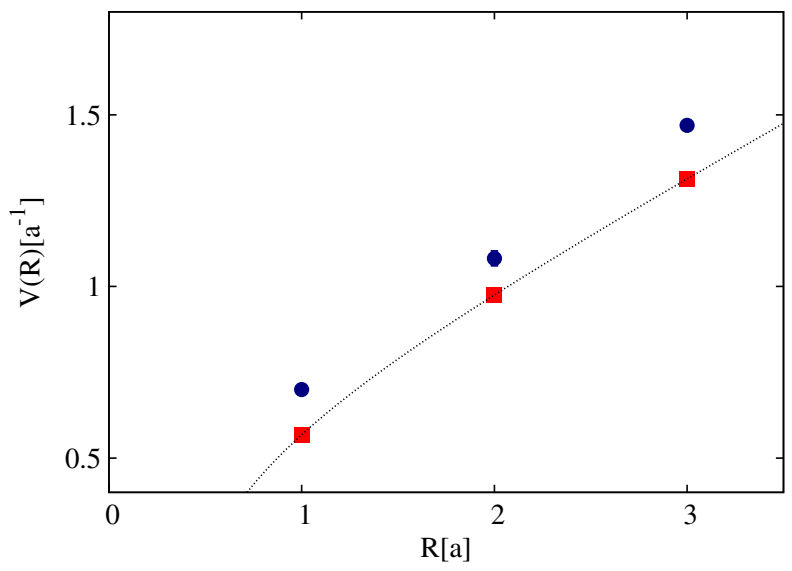

FIG. 5 (color online). The lattice QCD result (circles) of the interquark potential $V^{P}(R)$ after removing low-lying Dirac modes below the IR cutoff $\Lambda_{\mathrm{IR}}=0.5 a^{-1}$. Squares denote the original interquark potential. The potential is almost unchanged even after removing the low-lying Dirac modes, apart from an irrelevant constant. 

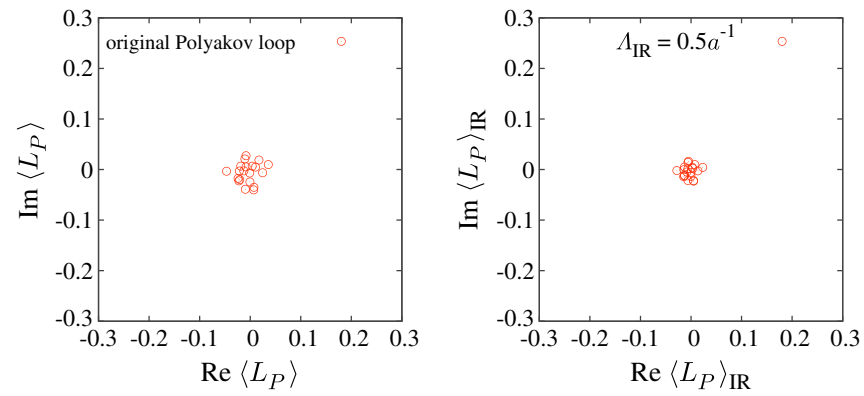

FIG. 6 (color online). The scatter plots of the Polyakov loop. The left panel shows the original Polyakov loop $\left\langle L_{P}\right\rangle$. The right panel shows the Polyakov loop $\left\langle L_{P}\right\rangle_{\mathrm{IR}}$ after cutting off the lowlying Dirac modes below the IR cutoff $\Lambda_{\mathrm{IR}}=0.5 a^{-1}$.

On the potential argument, we comment on the nonlocality stemming from the Dirac-mode projection, which makes the link variable extended and makes the potential meaning vague. This nonlocality appears hypercubic symmetrically in the four-dimensional space-time, and its effect would be maximal for the IR-cut case. As a whole, such a nonlocality makes the potential flat, because of the spatial averaging. (As an extreme example, the "potential" between wall-like sources is completely flat.) However, our obtained potential is almost the same as the original confining one, in spite of the possible flattening effect by the nonlocality. Therefore, regardless of the nonlocality, the confinement is kept after cutting off the low-lying Dirac modes (Since no flattening effect is observed in this projection, the nonlocality effect would not be significant, at least for the argument of confinement).

As another way to clarify the confinement on the periodic lattice, we also investigate the Polyakov loop $\left\langle L_{P}\right\rangle \equiv$ $\left\langle\operatorname{tr} \prod_{t=1}^{L} U_{4}(\vec{x}, t)\right\rangle / 3$ and the center $Z_{3}$-symmetry [5] in terms of the Dirac-mode projection. The Polyakov loop $\left\langle L_{P}\right\rangle$, which is usually used at finite temperature, can also be applied to our temporally periodic system on the link variable, and it physically relates to the quark singleparticle energy and the $Z_{3}$-symmetry [5]. Note that the nonlocality effect is less significant for the Polyakov loop $\left\langle L_{P}\right\rangle$ or the quark single-particle energy.

Now, we calculate the Polyakov loop with cutting off of the low-lying Dirac modes,

$$
\left\langle L_{P}\right\rangle_{\mathrm{IR}} \equiv \frac{1}{3} \frac{1}{V}\left\langle\operatorname{Tr}\left(\prod_{k=1}^{L} \hat{U}_{4}^{P}\right)\right\rangle=\frac{1}{3} \frac{1}{V}\left\langle\operatorname{Tr}\left\{\left(\hat{U}_{4}^{P}\right)^{L}\right\}\right\rangle,
$$

and its scatter plot, using the same lattice $\left(6^{4}, \beta=5.6\right)$ and the same IR cutoff $\Lambda_{\mathrm{IR}}=0.5 a^{-1}$. In the use of the full Dirac modes, i.e., $\hat{P}=1,\left\langle L_{P}\right\rangle_{\mathrm{IR}}$ coincides with $\left\langle L_{P}\right\rangle$. We show in Fig. 6 the scatter plot of the Polyakov loop $\left\langle L_{P}\right\rangle_{\mathrm{IR}}$ after cutting off the low-lying Dirac modes below $\Lambda_{\mathrm{IR}}=$ $0.5 a^{-1}$. We find that the IR-cut Polyakov loop $\left\langle L_{P}\right\rangle_{\mathrm{IR}}$ remains to be almost zero, i.e., $\left\langle L_{P}\right\rangle_{\mathrm{IR}} \simeq 0$, which corresponds to the $Z_{3}$-unbroken phase. In fact, even after removing the low-lying Dirac modes, which are responsible to chiral symmetry breaking, the single-quark energy is extremely large and the system is in the $Z_{3}$-unbroken confinement phase.

We also investigate the UV cut of Dirac modes in lattice QCD, using the UV-cut Dirac spectral density $\rho_{\mathrm{UV}}(\lambda) \equiv$ $\rho(\lambda) \theta\left(\Lambda_{\mathrm{UV}}-|\lambda|\right)$ with the UV cutoff $\Lambda_{\mathrm{UV}}=2 a^{-1} \simeq$ $1.6 \mathrm{GeV}$. In this case, unlike the IR cut, the chiral condensate is almost unchanged, and chiral symmetry breaking is almost kept. We show in Fig. 7 the UV-cut Wilson loop and the corresponding interquark potential, after removing the UV Dirac modes. We find that the area-law behavior of the Wilson loop and the slope parameter $\sigma^{P}$ are almost unchanged by the UV cut of the Dirac modes. This result seems consistent with the pioneering lattice study of Synatschke-Wipf-Langfeld [17]: they found that the confinement potential is almost reproduced only with low-lying Dirac modes using the spectral sum of the Polyakov loop [16,35].

Furthermore, we examine "intermediate (IM) cut", where a certain part of $\Lambda_{1}<\left|\lambda_{n}\right|<\Lambda_{2}$ of Dirac modes is removed. Unfortunately, when the wide region of Dirac modes is removed, the statistical error becomes quite large for the Dirac-mode projected Wilson loop. Here, we remove the IM Dirac modes of $0.5-0.8\left[a^{-1}\right]$,
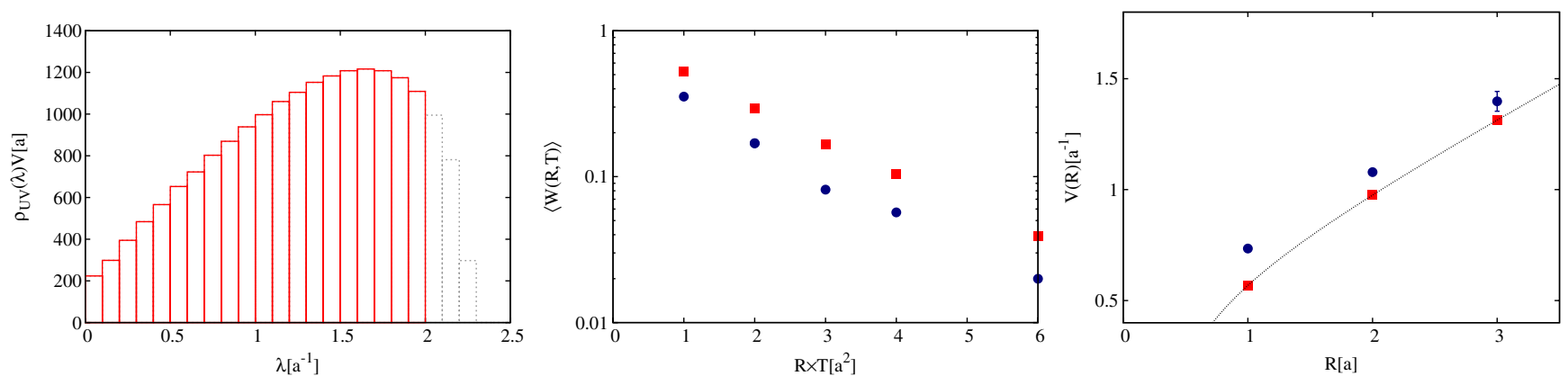

FIG. 7 (color online). (a) The UV-cut Dirac spectral density $\rho_{\mathrm{UV}}(\lambda) \equiv \rho(\lambda) \theta\left(\Lambda_{\mathrm{UV}}-|\lambda|\right)$ with the UV cutoff $\Lambda_{\mathrm{UV}}=2 a^{-1} \simeq$ $1.6 \mathrm{GeV}$. (b) The UV-cut Wilson loop $\operatorname{Tr} W^{P}(R, T)$ (circles) after removing the UV Dirac modes, plotted against $R \times T$. The slope parameter $\sigma^{P}$ is almost the same as that of the original Wilson loop (squares). (c) The corresponding UV-cut interquark potential (circles), which is almost unchanged from the original one (squares), apart from an irrelevant constant. 

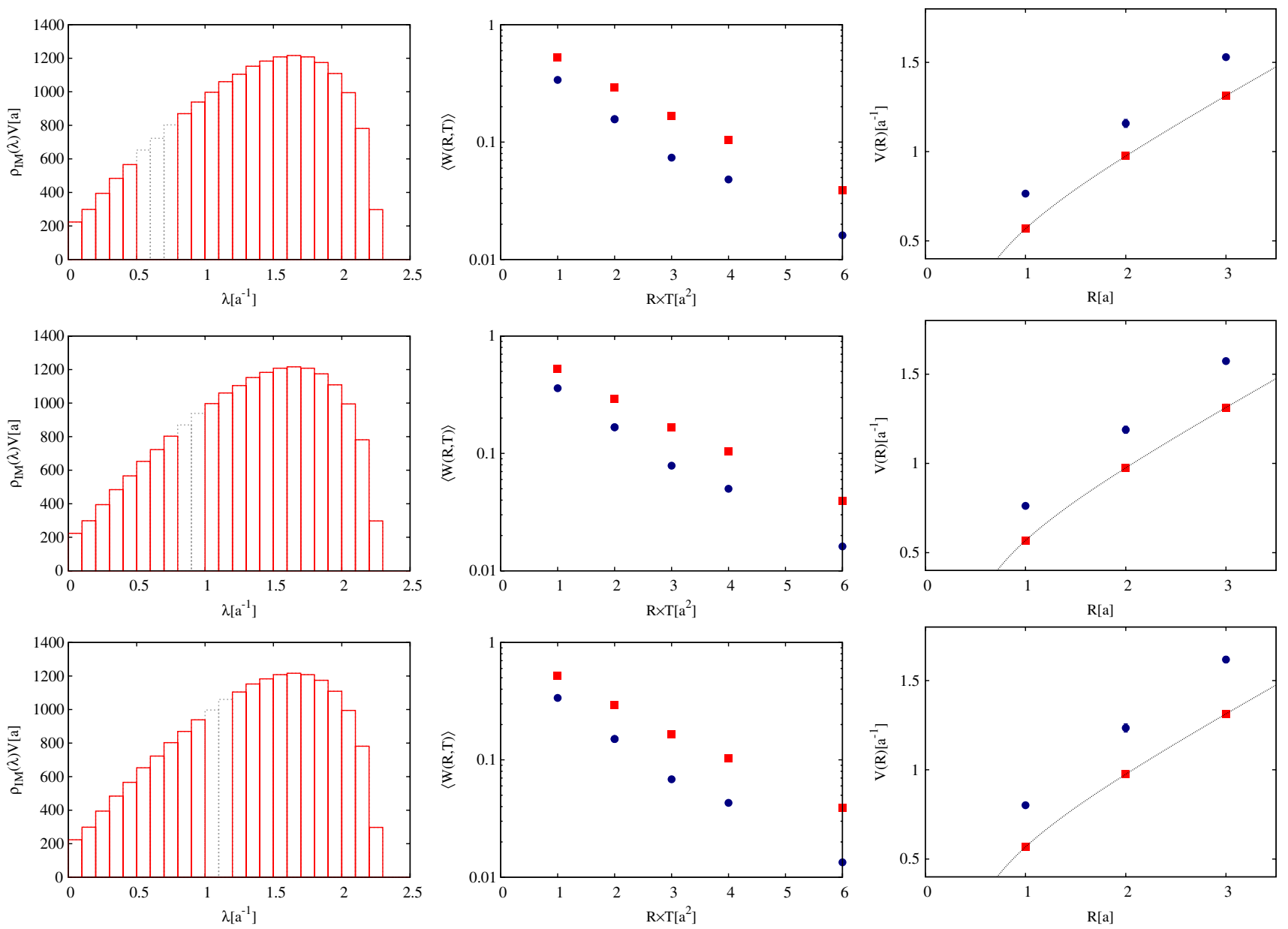

FIG. 8 (color online). The left panels show the intermediate (IM)-cut Dirac spectral density $\rho_{\text {IM }}(\lambda)$ : The IM Dirac modes of $0.5-0.8\left[a^{-1}\right]$ (top panel), 0.8-1.0[ $\left.a^{-1}\right]$ (middle panel), and 1.0-1.2[ $\left[a^{-1}\right]$ (bottom panel) are cut. The central panels show the IM-cut Wilson loop $\operatorname{Tr} W^{P}(R, T)$ (circles) after removing the IM Dirac modes, plotted against $R \times T$. For each case, the slope parameter $\sigma^{P}$ is almost the same as that of the original Wilson loop (squares). The right panels show the corresponding IM-cut interquark potential (circles), which is almost unchanged from the original one (squares), apart from an irrelevant constant.

$0.8-1.0\left[a^{-1}\right]$, and $1.0-1.2\left[a^{-1}\right]$, respectively, and investigate the corresponding IM-cut Wilson loop and the corresponding interquark potential in each case, as shown in Fig. 8. For each case, the area-law behavior of the Wilson loop and the slope parameter $\sigma^{P}$ are found to be almost unchanged by the IM cut of the Dirac modes.

Thus, from the above lattice QCD results, we conclude that there is no specific region of the Dirac modes responsible to the confinement in QCD, unlike the chiral symmetry breaking. Instead, we conjecture that the "seed" of confinement is distributed not only in low-lying Dirac modes, but also in a wider region of the Dirac-mode space.

\section{SUMMARY AND DISCUSSIONS}

We have developed a manifestly gauge-covariant expansion and projection using the eigenmode of the QCD Dirac operator $\not D=\gamma^{\mu} D^{\mu}$. With this method, we have performed a direct investigation of correspondence between confinement and chiral symmetry breaking in $\mathrm{SU}(3)$ lattice QCD on the $6^{4}$ periodic lattice at $\beta=5.6$ at the quenched level. We have found that the Wilson loop remains to obey the area law, and the slope parameter corresponding to the string tension, or confinement force, is almost unchanged, even after removing the low-lying Dirac modes, which are responsible to chiral symmetry breaking. We have also found that the Polyakov loop remains to be almost zero even without the low-lying Dirac modes, which indicates the $Z_{3}$-unbroken confinement phase. These results indicate that one-to-one correspondence does not hold between confinement and chiral symmetry breaking in QCD.

As a caution, we have used a coarse and small lattice, because of the technical difficulty to diagonalize the full Dirac operator. In particular, the box size of our lattice volume is about $1.5 \mathrm{fm}$. In fact, to be precise, this region we survey is the intermediate distance, of which confining behavior is rather important for the quark-hadron physics. 
To obtain more a definite conclusion, especially on the asymptotic confining behavior of the potential, it is desired to perform larger-volume lattice QCD calculations and to cut various wider regions of the Dirac modes, although it is technically quite difficult.

Our strategy is to investigate the relation between the nonperturbative properties of $\mathrm{QCD}$, by extracting or removing the essence of chiral symmetry breaking. This is similar to the demonstration of Abelian/monopole dominance $[9,12-14,21-25,36]$ or center/vortex dominance $[15,37,38]$ for nonperturbative properties. However, while the previous scenario has been done in a specific gauge, our new method is manifestly gauge invariant. In this analysis, we have carefully amputated only the "essence of chiral symmetry breaking" by cutting off the low-lying Dirac modes. Then, we have artificially realized the "confined but chiral restored situation" in QCD.

Recently, Lang and Schrock studied the hadron spectra after the cut of the low-lying Dirac modes [39]. Since the quark propagator is directly expressed with the Dirac operator $\not D$, the Dirac-mode projection is straightforward, and a complicated projection procedure is not necessary in such studies. In their study, although the confinement was not checked, the appearance of hadronic spectra seems to suggest the existence of the confinement force, even after cutting the low-lying Dirac modes.

Next, we comment on the possible relation among confinement, chiral symmetry breaking, and monopoles in QCD. There is a close relation between confinement and chiral symmetry breaking through the monopoles in the MA gauge [12-14]. The monopole would be essential degrees of freedom for most nonperturbative QCD: confinement [22], chiral symmetry breaking [13,14], and instantons [23]. In fact, removing the monopole would be "too fatal" for the nonperturbative properties, so that nonperturbative QCD phenomena are simultaneously lost by their cut. On the approximate coincidence of the critical temperatures of deconfinement and chiral restoration, a large change of monopoles may lead to both phase transitions [13], since the global connection of the monopole current seems to be largely changed around the QCD phase transition [24].

As for the recent finite-temperature QCD analysis, a lattice QCD group has reported a certain difference between the "critical temperatures" of deconfinement and chiral restoration, which are determined by the susceptibility peak of the Polyakov loop and chiral condensate, respectively [40]. This may also be indirect evidence of "confinement $\neq$ chiral symmetry breaking" in QCD.

Next, we briefly discuss the role of low-lying Dirac modes in the viewpoint of instantons in QCD. The Dirac zero-mode associated with an instanton is localized around it [11]. However, the localized objects are hard to contribute to the large-distance phenomenon of confinement, although such low-lying Dirac modes contribute to chiral symmetry breaking. Recall that instantons contribute to chiral symmetry breaking, but do not directly lead to confinement [11]. Then, as a thought experiment, if only instantons can be carefully removed from the QCD vacuum, confinement properties would be almost unchanged, but the chiral condensate is largely reduced, and accordingly some low-lying Dirac modes disappear. Thus, in this case, confinement is almost unchanged, in spite of the large reduction of low-lying Dirac modes.

If the relation between confinement and chiral symmetry breaking is not one-to-one in QCD, richer phase structure is expected in QCD. For example, the phase transition point can be different between deconfinement and chiral restoration in the presence of strong electromagnetic fields, because of their nontrivial effect on chiral symmetry [41]. It is also interesting to investigate the similar analysis at finite temperatures in lattice QCD. The full QCD calculation in this direction is also an interesting subject.

\section{ACKNOWLEDGMENTS}

The authors thank Professor K. Langfeld and Dr. T. Misumi for useful comments. H. S. is supported in part by the Grant for Scientific Research [(C) No. 23540306, Priority Areas "New Hadrons" (E01:21105006)] and T. I. is supported by Grant-in-Aid for JSPS Fellows (No. 23752), from the Ministry of Education, Culture, Science and Technology (MEXT) of Japan. This work is supported by the Global COE Program, "The Next Generation of Physics, Spun from Universality and Emergence." The lattice QCD calculations are done on NEC SX-8R at Osaka University.
[1] Y. Nambu and G. Jona-Lasinio, Phys. Rev. 122, 345 (1961); 124, 246 (1961).

[2] K. G. Wilson, Phys. Rev. D 10, 2445 (1974).

[3] J. B. Kogut and L. Susskind, Phys. Rev. D 11, 395 (1975).

[4] M. Creutz, Phys. Rev. Lett. 43, 553 (1979); Phys. Rev. D 21, 2308 (1980).

[5] H. J. Rothe, Lattice Gauge Theories (World Scientific, Singapore, 2005), and its references.
[6] M. Creutz, Quarks, Gluons and Lattices (Cambridge University Press, Cambridge, 1985); Acta Phys. Slovaca 61, 1 (2011), and its references.

[7] Y. Nambu, Phys. Rev. D 10, 4262 (1974); G. 't Hooft, in Proceedings of High Energy Physics (Editrice Compositori, Bologna, 1976)S. Mandelstam, Phys. Rep. 23, 245 (1976)..

[8] T. Banks and A. Casher, Nucl. Phys. B169, 103 (1980). 
[9] G. 't Hooft, Nucl. Phys. B190, 455 (1981).

[10] J. M. Cornwall, Phys. Rev. D 26, 1453 (1982).

[11] M. Shifman, Instantons in Gauge Theories (World Scientific, Singapore, 1994), and its references.

[12] H. Suganuma, S. Sasaki, and H. Toki, Nucl. Phys. B435, 207 (1995); S. Sasaki, H. Suganuma, and H. Toki, Prog. Theor. Phys. 94, 373 (1995); S. Umisedo, H. Suganuma, and H. Toki, Phys. Rev. D 57, 1605 (1998).

[13] O. Miyamura, Phys. Lett. B 353, 91 (1995).

[14] R. M. Woloshyn, Phys. Rev. D 51, 6411 (1995).

[15] R. Hollwieser, M. Faber, J. Greensite, U. M. Heller, and S. Olejnik, Phys. Rev. D 78, 054508 (2008).

[16] E. Bilgici, F. Bruckmann, C. Gattringer, and C. Hagen, Phys. Rev. D 77, 094007 (2008).

[17] F. Synatschke, A. Wipf, and K. Langfeld, Phys. Rev. D 77, 114018 (2008).

[18] J. M. Cornwall, Phys. Rev. D 83, 076001 (2011).

[19] J. M. Cornwall, J. Papavassiliou, and D. Binosi, The Pinch Technique and its Applications to Non-Abelian Gauge Theories (Cambridge University Press, Cambridge, 2011) and its references.

[20] F. Karsch, Lect. Notes Phys. 583, 209 (2002), and its references.

[21] A. S. Kronfeld, G. Schierholz, and U.-J. Wiese, Nucl. Phys. B293, 461 (1987); A. S. Kronfeld, M. L. Laursen, G. Schierholz, and U.-J. Wiese, Phys. Lett. B 198, 516 (1987).

[22] J. D. Stack, S. D. Neiman, and R. J. Wensley, Phys. Rev. D 50, 3399 (1994).

[23] H. Suganuma, A. Tanaka, S. Sasaki, and O. Miyamura, Nucl. Phys. B, Proc. Suppl. 47, 302 (1996).

[24] S.-I. Kitahara, Y. Matsubara, and T. Suzuki, Prog. Theor. Phys. 93, 1 (1995).

[25] K. Amemiya and H. Suganuma, Phys. Rev. D 60, 114509 (1999); H. Suganuma, K. Amemiya, H. Ichie, and A. Tanaka, Nucl. Phys. A670, 40 (2000).

[26] H. Suganuma, S. Gongyo, T. Iritani, and A. Yamamoto, Proc. Sci., QCD-TNT-II (2011) 044.
[27] A. Yamamoto and H. Suganuma, Phys. Rev. Lett. 101, 241601 (2008); Phys. Rev. D 79, 054504 (2009).

[28] A. Yamamoto and H. Suganuma, Phys. Rev. D 81, 014506 (2010); Proc. Sci., LAT2010 (2010) 294; A. Yamamoto, Nucl. Phys. B844, 115 (2011).

[29] T. Iritani, H. Suganuma, and H. Iida, Phys. Rev. D 80, 114505 (2009); T. Iritani and H. Suganuma, Phys. Rev. D 83, 054502 (2011).

[30] F. Bruckmann and E.-M. Ilgenfritz, Phys. Rev. D 72, 114502 (2005).

[31] M.F. Atiyah and I. M. Singer, Ann. Math. 87, 484 (1968).

[32] E. Anderson et al., LAPACK Users' Guide (Society for Industrial and Applied Mathematics, Philadelphia, 1999), 3rd ed.

[33] K. Nakamura et al. (Particle Data Group), J. Phys. G 37, 075021 (2010)..

[34] T. T. Takahashi, H. Suganuma, Y. Nemoto, and H. Matsufuru, Phys. Rev. D 65, 114509 (2002).

[35] C. Gattringer, Phys. Rev. Lett. 97, 032003 (2006); F. Bruckmann, C. Gattringer, and C. Hagen, Phys. Lett. B 647, 56 (2007).

[36] Z. F. Ezawa and A. Iwazaki, Phys. Rev. D 25, 2681 (1982); Phys. Rev. D 26, 631 (1982).

[37] L. Del Debbio, M. Faber, J. Greensite, and S. Olejnik, Phys. Rev. D 55, 2298 (1997).

[38] T. G. Kovacs and E. T. Tomboulis, Phys. Rev. D 57, 4054 (1998).

[39] C. B. Lang and M. Schrock, Phys. Rev. D 84, 087704 (2011); Proc. Sci., L AT2011 (2011) 111; L. Ya Glozman, C. B. Lang, and M. Schrock, Phys. Rev. D 86, 014507 (2012).

[40] Y. Aoki, Z. Fodor, S. D. Katz, and K. K. Szabo, Phys. Lett. B 643, 46 (2006).

[41] H. Suganuma and T. Tatsumi, Ann. Phys. (N.Y.) 208, 470 (1991); Prog. Theor. Phys. 90, 379 (1993). 